\title{
Towards Programmable Optical Network Front-end Devices Using Silicon Photonics (1525090-Y1)
}

\section{SHAYAN MOOKHERJEA}

Department of Electrical and Computer Engineering, University of California, San Diego, MC 0407 La Jolla CA 92093-0407 USA

Email:smookher@ucsd.edu

Summary of a Project Outcomes report of research funded by the U.S. National Science Foundation under Project Number 1525090 (Year 1). We study how the performance and utility of high-bandwidth, energy-efficient communication networks can be improved by enabling programmability and user-defined tunability in the optical front-ends using silicon photonics.

\section{Introduction}

In traditional computer system architecture, a system or host incorporates several functional elements, such as a computing process, memory and networking. Under the disaggregation concept, a network collocates some of the various computing, memory, storage, and networking resources for higher efficiency, to enable statistical multiplexing, greater operator control and software-defined networking. For large networks, this may imply at the physical level that common resources will be pooled e.g., racks of storage, or trays of processors, which makes an efficient, high-bandwidth interconnect technology essential [1], [2]. The concept is shown in Figure 1.
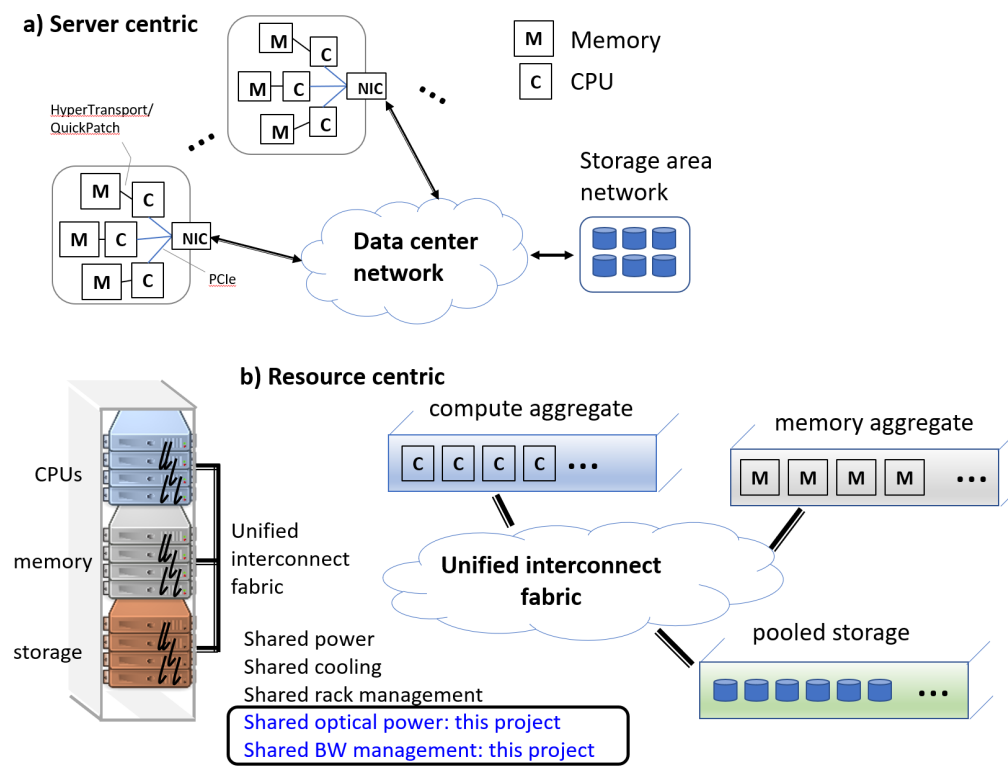

Figure 1 Comparison of traditional server-centric network architecture, and the disaggregated resource-centric architecture, which has several benefits including some (highlighted in blue) which become newly possible when optics (and, specifically, electronically-controlled integrated silicon photonics) is used as the interconnect technology. 
Disaggregation replaces a traditional model in which hosts or servers are the essential building block, and instead organizes by functionality. One possible approach is to rely on low-latency, low energy-cost, scalable interconnect technology to make this possible. Benefits of disaggregation depend on quickly and dynamically pooling resources needed to execute a particular task. But silicon photonic active cables provide a fixed bandwidth, fixed energy cost medium with the transmitter and receiver (some of the most energy consuming parts) built into each end of the cable. This is more restrictive than radiofrequency $(\mathrm{RF})$ communications or electronic interconnects, for example. In a silicon photonic cable, the only road to efficiency is through electronic components such as serializers and de-serializers, but these are already the most energy-hungry components, and their numbers would greatly increase, should disaggregation introduce several new classes of traffic flows with different bandwidth and latency requirements. To address this problem, we propose studying how to enable variable bandwidth control (available in RF and electronic links) in the optical channel using a physical-layer approach in silicon photonics, and also overlaying (logically) a distribution-network-style layer for node-state monitoring and state-variable control on top of the data network fabric.

\section{Goals and Strategy}

As a quantitative example, in Table 1 we calculate the utilization and (energy) cost of conventional optically-interconnected architecture versus a disaggregated architecture. For the former, we assume fixed bandwidth, fixed laser power links, and for the latter, we assume that these resources can be varied over 3 orders of magnitude by a central controller in response to the traffic matrix. In both cases, we assume for modeling purposes a power-law distribution of traffic flows with exponent 2.05 , link-availability requirement of $99.5 \%$ and an energy cost of $5 \mathrm{pJ} / \mathrm{bit}$ for $100 \mathrm{Gbit} / \mathrm{s}$ silicon photonic "active cables". As Table 1 shows, disaggregation can greatly improve link utilization by nearly a factor of 10 .

Table 1. Calculations of latency, utilization and energy cost.

\begin{tabular}{|l|c|c|c|c|}
\hline $\begin{array}{l}\text { Architecture } \\
\text { (idealized) }\end{array}$ & \# nodes $^{\text {(a) }}$ & $\begin{array}{c}\text { Datacenter fabric } \\
\text { latency }\end{array}$ & Utilization $^{(\mathbf{c}) \text { d) }}$ & $\begin{array}{c}\text { Energy cost } \\
\text { (interconnects } \\
\text { only }^{(\mathrm{e})} \text { ) }\end{array}$ \\
\hline Conventional & $1 \mathrm{E} 5$ & $14 \mu \mathrm{s}$ & $26 \%$ & $50 \mathrm{~kW}$ \\
\hline Disaggregated & $1 \mathrm{E} 5$ & $2.3 \mu \mathrm{s}$ & $>90 \%$ & $6.7 \mathrm{~kW}$ \\
\hline
\end{tabular}

(a) Assumption; 3-tier fat tree architecture using switches with the same port counts on all levels.

(b) Link length assumed to be $500 \mathrm{~m}$ (uniform). Amount of time taken to traverse the network.

(c) Based on calculation of expected (mean) size of traffic flow / peak cable capacity over all links; the probability of flows exceeding the link bandwidth assumed to be $0.5 \%$. 
(d) Data overhead due to disaggregated additional control/monitoring information assumed to be $<$ $10 \%$.

(e) Assuming $5 \mathrm{pJ} / \mathrm{bit}$ data cost for active optical cable (including driver and detector circuits).

Since the late 1980's, researchers have been advocating the benefits of silicon photonics as a platform for integrated optics devices. At about the same time, the Semiconductor Industry Association (SIA) warned of the impending performance limits of CPUs and bottleneck in interconnect delay as technology continued to improve. By 2006, silicon optical technology for $2.5 \mathrm{Gbit} / \mathrm{s}$ signaling, capable of using 150 Watts of power to provide $10 \mathrm{~km}$ interconnects across 8 metal layers was developed (conceptually). Indeed, optical interconnects are being proposed for a wide range of computer systems, ranging from traces within a printed circuit board which connect multiple CPU or GPU cores, to "active cables" with a form factor that resembles that of a USB cable, and can interconnect consumer devices such as ultra-high-definition televisions to network or storage appliances. The biggest near-term application of optical interconnects is expected to be in Data Center Networks (DCN's), enabling energy-effective scaling to many millions of nodes, and eventually to ExaScale systems.

In this project, we will attempt to show that silicon photonics can indeed be compatible with disaggregation concepts if:

(1) We compensate for the separation of previously proximal blocks by using optics to carry control and monitoring signals between hosts and the controller unit, wherever it may be located on the network. This will reduce latency significantly compared to electronic interconnects. Other than compute, memory and storage aggregates (see Fig. 1), other physical resources may also be aggregated and delivered e.g., laser power. Optical fiber is a suitable technology for decoupling the inherent distance-energy-latency tradeoffs of electronic wires.

(2) We compensate for the increased number of logical interfaces without increasing the number of fibers if we can co-transmit the new signals required for disaggregation over low-bandwidth, spectrally-separated "lambdas" on the same fibers as those which carry data (see Fig. 2). This may not be the only solution, but is elegant in silicon photonics, and leverages strengths of this platform (high bandwidth, small footprint, ability to realize complex spectral filtering, incorporation of monitoring photodiodes and baseband electronic amplifiers, etc.) For example, we will study how to extract and insert variable bandwidth slices of spectral content from a common "bus" fiber without disturbing other channels.

(3) We permit the adaptability of physical resources e.g., varying optical powers or link bandwidths (sometimes called "flexgrid", see Fig. 2a) on demand in order to allow software-defined or statistical traffic multiplexing. Overprovisioned optical links waste laser power and present a photonic 
equivalent of today's "dark silicon" problem whereby a significant fraction of the hardware resources must remain under-utilized because of energy cost constraints. While it is relatively simple to meet the bandwidth requirements of current-generation modulators, emerging devices are likely to place a higher burden on the filters [3].

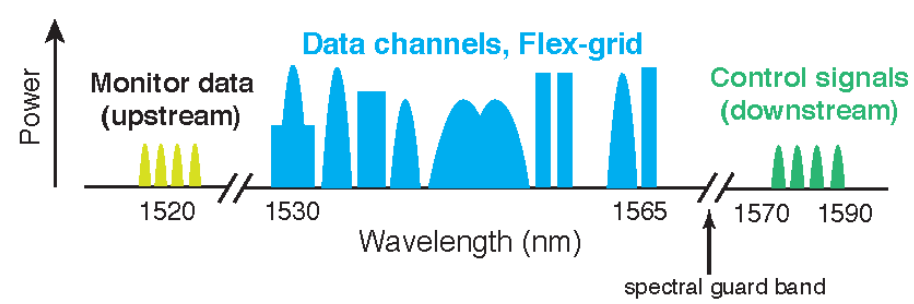

b

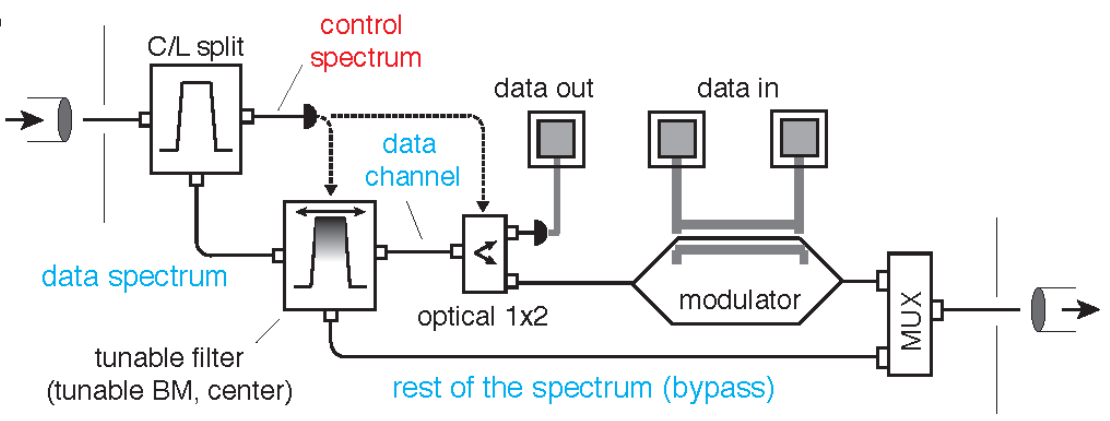

Figure 2 key underpinning (PHY level) concept: monitor and control signals are added to / removed from the data-carrying channels over the same optical fiber and over the same physical connector ports to overcome latency-distance and port-count limitations. In this cartoon, the data spectral band is densely packed with different types of data, thus forcing the control and monitoring signals to reside out-of-band. In most real networks today, the C-band utilization is quite sparse, leaving "holes" in the spectrum which could be used by intelligent modules as long as they are able to tune between the ITU-T grid lines

\section{Progress and Impact}

As shown in Fig. 2b, the first functionality we need to demonstrate was the ability to take a standard multi-channel optical wavelength-division multiplexed data stream - which is typically on the ITU-T grid of frequencies, and insert and remove other wavelengths "off-grid" which can be used for distributing control signals. (This is the "Flex-Grid" concept described in our proposal). We have successfully accomplished this for a point-to-point transmission. To do this, we designed a chip using our library of silicon photonics components, had the chip manufactured as part of a multi-project wafer process at Sandia National Labs, designed a printed circuit board and had the received chips die-on-board assembled and wire-bonded by a local assembly company (see Fig. 3), and then tested it. The control signals in this demonstration were sent by typing commands on a computer (within Matlab, driving signals through a D/A module). At this time, the PCB and control signals are all low-speed $(<1 \mathrm{MHz})$. 


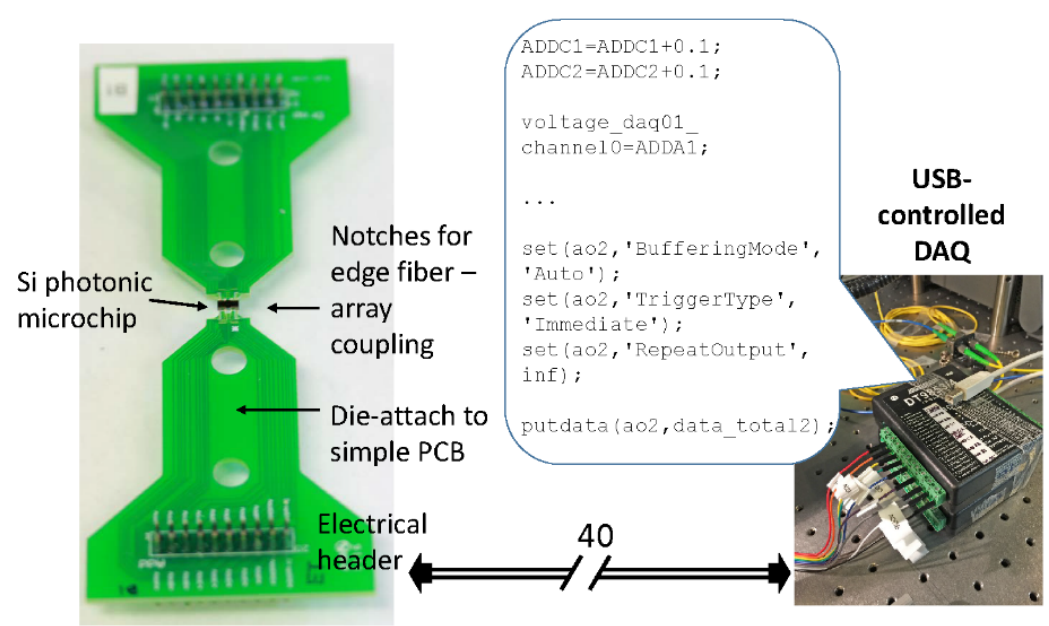

Figure 3 A chip was designed, fabricated and wire-bonded for testing. Commands that affected the behavior of the chip (essentially, tuning spectral filters and power levels through optical power control) were sent from a computer through DAC boards.

The typical device architecture that we use is a tunable optical filter, that is based on the coupled-resonator optical waveguide concept [4], [5]. There is interesting physics in how light propagates in such a structure [6] (based on the coupling of optical microresonators [7]) which affects the dispersion characteristics of the channels [8]-[10]. Although we have mostly tested our devices at lower optical powers, there are effects that should be considered at higher optical power levels, if the devices are placed close to the head-end of the network and lasers are run at elevated power levels [11]-[14]. We also use single microring resonators as filters when the additional performance of coupled resonators is not necessary [15], [16].

Next, we assembled a 4-data-channel testbed and used it to demonstrate that the chip shown in Fig. 3 could be used to add and drop channels from a wavelength-division multiplexed communications channel, as shown in Fig. 4. These initial experiments were performed using unmodulated laser light, from individual lasers tuned to specific wavelengths, and then multiplexed onto a common waveguide, which was coupled to an optical fiber. This was performed using multiple banks of individually-addressed, thermally-tunable optical adddrop filters on a common bus waveguide. The dispersion of the coupler between the filter and the bus waveguide is especially important to achieve wideband performance [17]-[19]. Further details and tests with real modulated data will be presented in subsequent reports.

Another set of challenges involves how to fabricate, test and improve devices, especially those based on microresonators, which are notoriously sensitive to fabrication imperfections [20]. Here, infrared imaging techniques and postfabrication compensation methods may be useful [21]-[23]. 
External optical amplification was used to compensate for the waveguide-fiber coupling losses, which can be improved in the future. The CROW architecture is also amenable to tuning using current injection and optical gain, which may be useful for compensating the optical insertion loss [24], [25].

At this time, neither the networking architecture nor the device design has fully stabilized, and both continue to evolve. However, industry is picking up these research trends ["Nokia Bell Labs reboots bandwidth variable transmitter" [optics.org newsdesk, 03 Feb 2016].
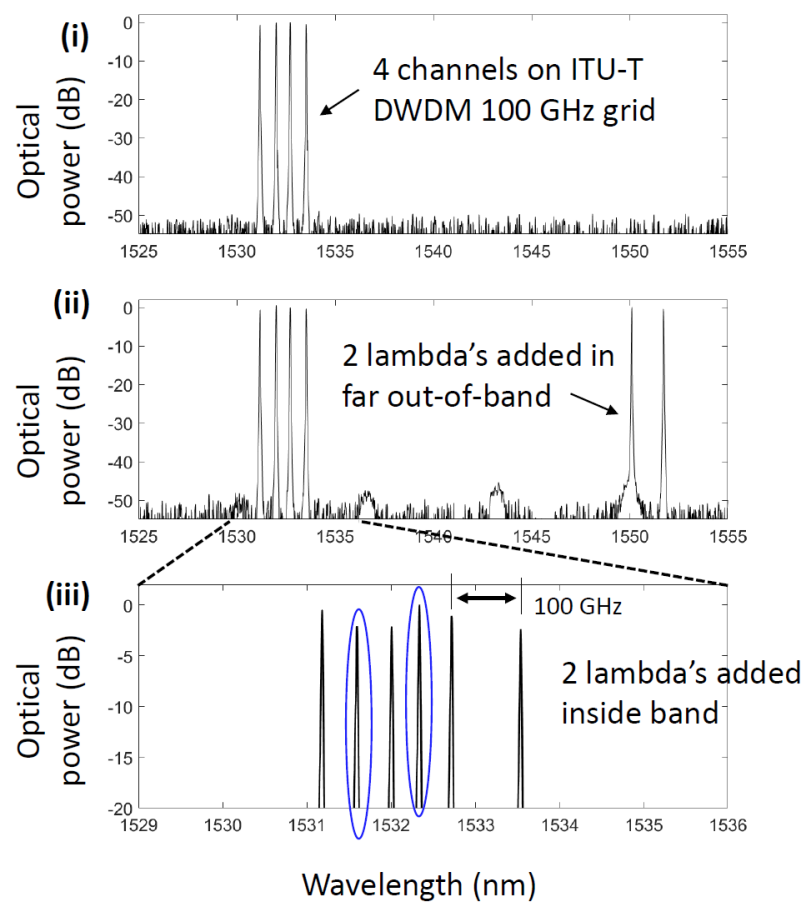

Figure 4 Successive spectrum images of (i) a four ITU-T $100 \mathrm{GHz}$ DWDM grid lambdas going
through the chip (ii) The goal of the test was to add "off-the-grid" two control signals, at remote
wavelengths as shown in the original concept and demonstrated here. (iii) As an improved
demonstration, we were able, by adjusting voltages (of thermal tuners) and reconfiguring silicon
photonics optical components on the chip, to insert the new channels "off-the-grid" between the
existing lambdas. This may allow better spectral utilization in current-usage real network
implementations, which are quite far from being spectrally densely filled (unlike the cartoon in
Fig. 2a)

A speculative track of this project is to study whether the same technology used to implement filtering and add/drop functionality in the realm of classical communications can also be used to implement quantum side channels over the same optical fiber. Preliminary results suggest that silicon photonics can be used for efficient wavelength conversion (using four-wave mixing) integrated with filtering [26], and that devices can also be used for generating entangled photon pairs (using spontaneous four-wave mixing) [27]-[29]. The area of nonlinear optics and dispersive propagation is rich in physics [30], [31], and 
can lead to a deeper understanding of how to control light propagation in tightly-confined microstructures.

\section{Open-Access Reporting Initiative}

PRAISE: This open-access document is provided in support of our PRAISE (Public Report of Activities, Impact and Subsequent Extensions) initiative. What is it? An open-access document shared with the public which describes the research outcomes of publicly-funded projects such as those funded by the U.S. NSF (National Science Foundation).

\section{References}

[1] S. Han, N. Egi, A. Panda, S. Ratnasamy, G. Shi, and S. Shenker, "Network support for resource disaggregation in next-generation datacenters," in Proceedings of the Twelfth ACM Workshop on Hot Topics in Networks, College Park Maryland, Nov. 2013, pp. 1-7. doi: 10.1145/2535771.2535778.

[2] J. Lee et al., "Application-driven bandwidth guarantees in datacenters," in Proceedings of the 2014 ACM conference on SIGCOMM, Chicago Illinois USA, Aug. 2014, pp. 467-478. doi: 10.1145/2619239.2626326.

[3] X. Wang, P. O. Weigel, J. Zhao, M. Ruesing, and S. Mookherjea, "Achieving beyond-100-GHz large-signal modulation bandwidth in hybrid silicon photonics Mach Zehnder modulators using thin film lithium niobate," APL Photonics, vol. 4, no. 9, p. 096101, Sep. 2019, doi: 10.1063/1.5115243.

[4] M. L. Cooper et al., "Waveguide dispersion effects in silicon-on-insulator coupled-resonator optical waveguides," Opt. Lett., vol. 35, no. 18, p. 3030, Sep. 2010, doi: 10.1364/OL.35.003030.

[5] M. L. Cooper et al., "235-ring Coupled-Resonator Optical Waveguides," in Conference on Lasers and Electro-Optics 2010, San Jose, California, 2010, p. CTUHH3. doi: 10.1364/CLEO.2010.CTuHH3.

[6] S. Mookherjea and A. Yariv, "Optical pulse propagation in the tightbinding approximation," Opt. Express, vol. 9, no. 2, p. 91, Jul. 2001, doi: 10.1364/OE.9.000091.

[7] S. Mookherjea, "Spectral characteristics of coupled resonators," J. Opt. Soc. Am. B, vol. 23, no. 6, p. 1137, Jun. 2006, doi: 10.1364/JOSAB.23.001137.

[8] S. Mookherjea and A. Yariv, "Pulse propagation in a coupled resonator optical waveguide to all orders of dispersion," Phys. Rev. E, vol. 65, no. 5, p. 056601, Apr. 2002, doi: 10.1103/PhysRevE.65.056601. 
[9] S. Mookherjea, D. S. Cohen, and A. Yariv, "Nonlinear dispersion in a coupled-resonator optical waveguide," Opt. Lett., vol. 27, no. 11, p. 933, Jun. 2002, doi: 10.1364/OL.27.000933.

[10] S. Mookherjea, "Dispersion characteristics of coupled-resonator optical waveguides," Opt. Lett., vol. 30, no. 18, p. 2406, Sep. 2005, doi: 10.1364/OL.30.002406.

[11] S. Mookherjea and M. A. Schneider, "The nonlinear microring adddrop filter," Opt. Express, vol. 16, no. 19, p. 15130, Sep. 2008, doi: 10.1364/OE.16.015130.

[12] S. Mookherjea and A. Yariv, "Kerr-stabilized super-resonant modes in coupled-resonator optical waveguides," Phys. Rev. E, vol. 66, no. 4, p. 046610, Oct. 2002, doi: 10.1103/PhysRevE.66.046610.

[13] J. R. Ong et al., "Low-power continuous-wave four-wave mixing in silicon coupled-resonator optical waveguides," Opt. Lett., vol. 36, no. 15, pp. 2964-2966, 2011.

[14] S. Mookherjea and A. Yariv, "Optical pulse propagation and holographic storage in a coupled-resonator optical waveguide," Phys. Rev. $E$, vol. 64, no. 6, p. 066602 , Nov. 2001, doi: 10.1103/PhysRevE.64.066602.

[15] R. Aguinaldo et al., "Wideband silicon-photonic thermo-optic switch in a wavelength-division multiplexed ring network," Opt. Express, vol. 22, no. 7, p. 8205, Apr. 2014, doi: 10.1364/OE.22.008205.

[16] R. Aguinaldo, Yiran Shen, and S. Mookherjea, "Large Dispersion of Silicon Directional Couplers Obtained via Wideband Microring Parametric Characterization," IEEE Photon. Technol. Lett., vol. 24, no. 14, pp. 1242-1244, Jul. 2012, doi: 10.1109/LPT.2012.2198639.

[17] M. L. Cooper and S. Mookherjea, "Numerically-assisted coupledmode theory for silicon waveguide couplers and arrayed waveguides," Opt. Express, vol. 17, no. 3, p. 1583, Feb. 2009, doi: 10.1364/OE.17.001583.

[18] S. Mookherjea and M. A. Schneider, "Avoiding bandwidth collapse in long chains of coupled optical microresonators," Opt. Lett., vol. 36, no. 23, p. 4557, Dec. 2011, doi: 10.1364/OL.36.004557.

[19] M. L. Cooper and S. Mookherjea, "Modeling of Multiband Transmission in Long Silicon Coupled-Resonator Optical Waveguides," IEEE Photon. Technol. Lett., vol. 23, no. 13, pp. 872-874, Jul. 2011, doi: 10.1109/LPT.2011.2141657.

[20] S. Mookherjea, J. R. Ong, X. Luo, and L. Guo-Qiang, "Electronic control of optical Anderson localization modes," Nature Nanotech, vol. 9, no. 5, pp. 365-371, May 2014, doi: 10.1038/nnano.2014.53.

[21] M. L. Cooper, G. Gupta, J. S. Park, M. A. Schneider, I. B. Divliansky, and S. Mookherjea, "Quantitative infrared imaging of silicon-on-insulator 
microring resonators," Opt. Lett., vol. 35, no. 5, p. 784, Mar. 2010, doi: 10.1364/OL.35.000784.

[22] Y. Shen, I. B. Divliansky, D. N. Basov, and S. Mookherjea, "Perfect set-and-forget alignment of silicon photonic resonators and interferometers," in Optical Fiber Communication Conference/National Fiber Optic Engineers Conference 2011, Los Angeles, California, 2011, p. PDPC3. doi: 10.1364/OFC.2011.PDPC3.

[23] S. Mookherjea and H. R. Grant, "High dynamic range microscope infrared imaging of silicon nanophotonic devices," Opt. Lett., vol. 37, no. 22, p. 4705, Nov. 2012, doi: 10.1364/OL.37.004705.

[24] S. Mookherjea, "Semiconductor coupled-resonator optical waveguide laser," Appl. Phys. Lett., vol. 84, no. 17, pp. 3265-3267, Apr. 2004, doi: 10.1063/1.1719278.

[25] S. Mookherjea, "Using gain to tune the dispersion relation of coupledresonator optical waveguides," IEEE Photon. Technol. Lett., vol. 18, no. 5, pp. 715-717, Mar. 2006, doi: 10.1109/LPT.2006.871144.

[26] J. R. Ong, R. Kumar, and S. Mookherjea, "Silicon microring-based wavelength converter with integrated pump and signal suppression," Opt. Lett., vol. 39, no. 15, p. 4439, Aug. 2014, doi: 10.1364/OL.39.004439.

[27] J. R. Ong and S. Mookherjea, "Quantum light generation on a silicon chip using waveguides and resonators," Opt. Express, vol. 21, no. 4, p. 5171, Feb. 2013, doi: 10.1364/OE.21.005171.

[28] M. Savanier, R. Kumar, and S. Mookherjea, "Optimizing photon-pair generation electronically using a $p-i-n$ diode incorporated in a silicon microring resonator," Appl. Phys. Lett., vol. 107, no. 13, p. 131101, Sep. 2015, doi: 10.1063/1.4932047.

[29] R. Kumar, M. Savanier, J. R. Ong, and S. Mookherjea, "Entanglement measurement of a coupled silicon microring photon pair source," Opt. Express, vol. 23, no. 15, p. 19318, Jul. 2015, doi: 10.1364/OE.23.019318.

[30] A. Ciattoni, B. Crosignani, S. Mookherjea, and A. Yariv, "Nonparaxial dark solitons in optical Kerr media," Opt. Lett., vol. 30, no. 5, p. 516, Mar. 2005, doi: 10.1364/OL.30.000516.

[31] B. Crosignani, A. Yariv, and S. Mookherjea, "Nonparaxial spatial solitons and propagation-invariant pattern solutions in optical Kerr media," Opt. Lett., vol. 29, no. 11, p. 1254, Jun. 2004, doi: 10.1364/OL.29.001254. 